\title{
Administration of Subcutaneous Buffered Lidocaine Prior to Breast Lymphoscintigraphy Reduces Pain Without Decreasing Lymph Node Visualization
}

\author{
Andrew S. Hawkins ${ }^{1}$, Don C. Yoo ${ }^{1}$, Jonathan S. Movson ${ }^{1}$, Richard B. Noto ${ }^{1}$, Kelly Powers ${ }^{2}$, and Grayson L. Baird ${ }^{1}$ \\ ${ }^{1}$ Department of Diagnostic Imaging, The Warren Alpert Medical School of Brown University, Rhode Island Hospital, Providence, \\ Rhode Island; and ${ }^{2}$ Alaska Radiology Associates, Anchorage, Alaska
}

Breast lymphoscintigraphy using ${ }^{99 m T c-s u l f u r ~ c o l l o i d ~(99 m T c-S C) ~}$ is well established in clinical practice for staging patients with breast carcinoma. Nearly all patients report having pain during the procedure. However, techniques used to minimize pain during breast lymphoscintigraphy are highly variable across institutions. Our study was to determine whether anesthetizing the skin with sodium bicarbonatehether-buffered lidocaine before performing breast lymphoscintigraphy reduced the pain experienced by the patients. The second objective of this study was to evaluate whether anesthetizing the skin with buffered lidocaine changed visualization of lymph nodes. Methods: This prospective, patient-masked and randomized study involved performing breast lymphoscintigraphy in a control group and experimental group of female breast cancer patients. The control group did not receive skin anesthetic before ${ }^{99 \mathrm{~m} T c-S C}$ injections, whereas the experimental group first underwent skin anesthesia with an injection of $2 \%$ sodium bicarbonate-buffered lidocaine. All patients were asked to rate their pain levels, using the National Institutes of Health pain scale, before the procedure and immediately after the injections. The change in pain from baseline was compared between the 2 groups. After the injections, scintigraphic imaging of the axilla was performed, and the number of axillary lymph nodes visualized was recorded. Results: No significant difference was found in preprocedural baseline pain from the control group, compared with the experimental group. There was a statistically significant difference in the increase in pain experienced during the procedure between the control group and the experimental group $(P=$ 0.009). There was no significant difference in the detection of lymph nodes between the control and experimental groups $(P=0.56)$. Conclusion: The results from our study indicate that injecting subcutaneous buffered lidocaine before intradermal injection of $99 \mathrm{mTC}$-SC for breast lymphoscintigraphy significantly decreases patient pain without interfering with lymph node visualization.

\footnotetext{
Received Jun. 19, 2014; revision accepted Sep. 18, 2014.

For correspondence or reprints contact: Don C. Yoo, Department of Diagnostic Imaging, The Warren Alpert Medical Scool of Brown University,

Rhode Island Hospital, Providence, RI 02818.

E-mail: dyoo@lifespan.org

Published online Oct. 23, 2014.

COPYRIGHT (C) 2014 by the Society of Nuclear Medicine and Molecular Imaging, Inc.
}

Key Words: breast; cancer; lymphoscintigraphy; anesthesia; lidocaine

J Nucl Med Technol 2014; 42:260-264

DOI: 10.2967/jnmt.114.144402

B reast lymphoscintigraphy using ${ }^{99 \mathrm{~m} T c-s u l f u r}$ colloid $\left({ }^{99 \mathrm{~m}} \mathrm{Tc}-\mathrm{SC}\right)$ is well established in clinical practice for staging patients with breast carcinoma. Intraoperative localization of the sentinel node using a $\gamma$ probe allows staging of the axilla while avoiding a full axillary dissection and its associated morbidity.

For any procedure, an inherent goal is to minimize discomfort while maximizing diagnostic yield. However, techniques used to minimize pain during breast lymphoscintigraphy are highly variable across institutions. The first objective of our study was to determine whether anesthetizing the skin with an injection of sodium bicarbonate-buffered lidocaine before performing breast lymphoscintigraphy reduced the amount of pain experienced by the patients. The second objective of this study was to evaluate whether the injection of sodium bicarbonate-buffered lidocaine decreased visualization of lymph nodes.

\section{MATERIALS AND METHODS}

\section{Patient Selection}

This prospective, patient-masked and randomized study was compliant with the Health Insurance Portability and Accountability Act. The institutional review board (IRB or equivalent) approved this study, and all subjects signed a written informed consent form. The eligibility requirements included patients $18 \mathrm{y}$ or older with a biopsy-proven breast cancer scheduled for sentinel lymph node biopsy and no known allergy to lidocaine. Over a 5-mo period between November 9, 2009, and April 10, 2010, 49 women who met the eligibility requirements and who agreed to participate in the study were randomized to either the control group (breast lymphoscintigraphy without lidocaine) or the experimental group (breast lymphoscintigraphy preceded by lidocaine injection). All injections were performed by 1 of 3 board-certified radiology attendings, all with fellowship training in nuclear medicine and more than $10 \mathrm{y}$ of experience performing breast lymphoscintigraphy. 
TABLE 1

NIH Pain Scale Gradations

\begin{tabular}{|c|c|}
\hline Gradation & Descriptor \\
\hline 0 & No pain \\
\hline $1-3$ & $\begin{array}{l}\text { Mild pain (nagging, annoying, interfering little } \\
\text { with ADLs) }\end{array}$ \\
\hline $4-6$ & Moderate pain (interferes significantly with ADLs) \\
\hline $7-10$ & Severe pain (disabling; unable to perform ADLs) \\
\hline \multicolumn{2}{|c|}{ ADLs $=$ activities of daily living. } \\
\hline
\end{tabular}

\section{Buffered Lidocaine Preparation}

Immediately before injection, the radiologist performing the injection withdrew $1 \mathrm{~mL}$ of sodium bicarbonate into a $5-\mathrm{mL}$ syringe capable of holding a total volume of $6 \mathrm{~mL}$. The remainder of the syringe was filled with $2 \%$ lidocaine.

\section{Injection Technique}

Breast lymphoscintigraphy was performed following our routine departmental protocol for the control group; because no placebo was used, this constitutes treatment as usual. Patients were not told whether they would receive the buffered lidocaine. Patients were asked to turn their head and not watch the injections being performed. The dose of ${ }^{99 \mathrm{~m}} \mathrm{Tc}-\mathrm{SC}$ was separated into two 3-mL syringes. The skin in the peritumoral or periareolar breast was cleansed with alcohol wipes. Two intradermal injections were then administered in the general region of the known breast neoplasm or in the periareolar location (at the 3 and 9 o'clock positions), depending on the preferences of the referring breast surgeon.

In the experimental group, patients were also asked to turn their head and not watch the injections being performed. After the skin was cleansed with alcohol wipes, the $6 \mathrm{~mL}$ of buffered lidocaine was administered subcutaneously (via 1 needle stick for peritumoral injections and 2 needle sticks for periareolar injections) before ${ }^{99 \mathrm{~m} T c-S C}$ injections were performed, using the same routine departmental protocol.

\section{Data Collection Technique}

All patients were asked to rate their pain levels before the procedure and immediately after the injections had been completed using the National Institutes of Health (NIH) pain scale (1). The NIH pain scale is graded from 0 to 10 , as shown in Table 1 . These values were recorded by a research assistant who was present throughout the procedure. Blood pressure and heart rates were recorded at the beginning of the patient encounter and after the injection. Averages of blood pressures and heart rates were calculated. A summary of the control group and experimental group characteristics is provided in Table 2.

\section{Imaging Parameters and Technique}

Approximately $30 \mathrm{~min}$ after breast lymphoscintigraphy, imaging was performed according to routine departmental breast imaging procedure. Anterior, lateral, and oblique spot planar images were obtained using a Philips Forte and Philips Skylight $\gamma$ camera.

\section{Statistical Methods}

Data were modeled before and after treatment by treatment group using SAS/GLIMMIX Statistical Software 9.3 (SAS Institute). Baseline differences were tested using SAS/NPAR1WAY and SAS/TTEST. Difference in lymph node visualization between conditions was tested using the Pearson $\chi^{2}$ test with SAS/FREQ. Statistical significance was defined, a priori, at the 0.05 level. In addition, follow-up multiple comparisons were tested using orthogonal linear contrasts with the Bonferroni method to adjust individual $P$ values.

\section{RESULTS}

For those in the control condition, average, median, and range of age were $59,55.5$, and $44-83 \mathrm{y}$, respectively; for those in the experimental lidocaine condition, average, median, and range of age were $63.8,65$, and $34-87 \mathrm{y}$, respectively. No difference in age was found between conditions $(P=0.16)$. No significant difference was found in preprocedural pain from the peritumoral-injection control group, compared with the lidocaine group at baseline $(P=0.59)$. In addition, no differences between the 2 conditions were detected at baseline for heart rate $(P=0.62)$ and systolic $(P=$ $0.26)$ and diastolic $(P=0.43)$ blood pressure. Average, median, and range of tumor diameters were 2.1, 1.5, and 0.4$12 \mathrm{~cm}$, respectively, for those in the control group versus $1.53,1.5$, and $0.4-4.0 \mathrm{~cm}$, respectively, for the lidocaine group, though this difference was not significant $(P=0.67)$.

The pre- and posttreatment pain by treatment condition was analyzed using a generalized mixed model with sandwich

TABLE 2

Patient Characteristics

\begin{tabular}{|c|c|c|c|}
\hline Characteristic & All & Control group & Experimental group \\
\hline Female patients $(n)$ & 49 & 24 (49\%) & 25 (51\%) \\
\hline \multicolumn{4}{|l|}{ Age } \\
\hline Range (y) & $34-87$ & $44-83$ & $34-87$ \\
\hline Mean (y) & 61.4 & 59 & 64 \\
\hline Mean blood pressure (systolic/diastolic) $(\mathrm{mm} / \mathrm{hg})$ & $162 / 82$ & $177 / 81$ & $148 / 83$ \\
\hline Mean heart rate (beats per min) & 79 & 77 & 80 \\
\hline Tumor grade* & 1.8 & 1.6 & 2.0 \\
\hline \multicolumn{4}{|l|}{ Tumor size $(\mathrm{cm})$} \\
\hline Range & $0.4-12$ & $0.4-12$ & $0.4-4.0$ \\
\hline Mean & 1.8 & 2.1 & 1.5 \\
\hline Total volume injected (SC and lidocaine if administered) & 3.61 & 1.1 & 6.2 \\
\hline
\end{tabular}




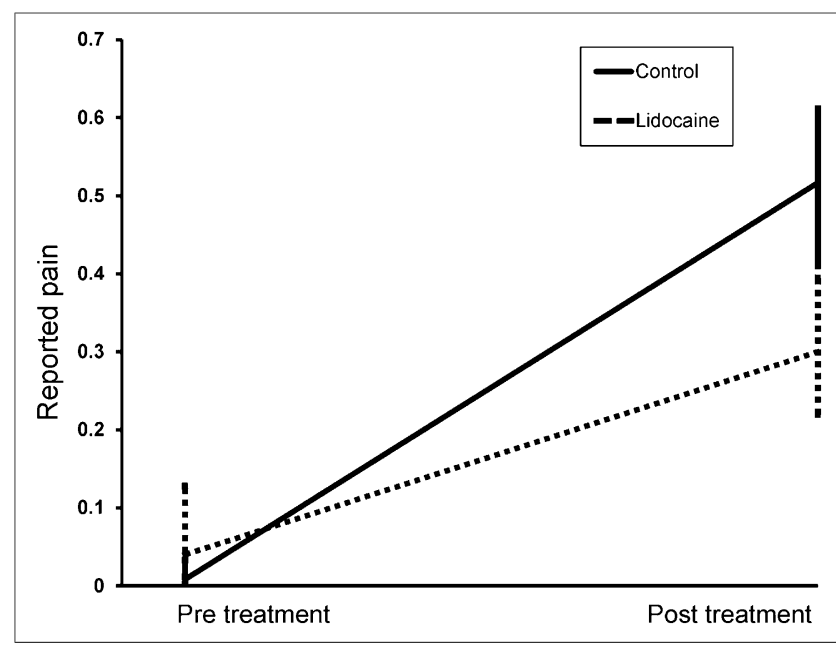

FIGURE 1. Reported pain before and after treatment.

estimation. A binomial link function was used because estimated confidence intervals fell outside of possible bounds (i.e., went below 0 ) when an identity link was used. A significant interaction effect with pre-/posttreatment and treatment condition was found $(P=0.009)$. Specifically, though both groups reported an increase in pain after the procedure relative to baseline, the lidocaine condition reported significantly less of an increase in pain, compared with the control group (Fig. 1; Table 3).

\section{Sentinel Lymph Node Detection}

Of the control group, 19 of 24 (79\%) had 1 or more axillary lymph nodes visualized, compared with 18 of 25 (72\%) for the experimental group. Five of $24(21 \%)$ of the control group patients and 7 of $25(28 \%)$ of the experimental group patients had no lymph node uptake visualized scintigraphically in the axilla at the time of imaging. There was no significant difference in the detection of lymph nodes between the control and experimental groups $(P=0.56)$ (Figs. 2 and 3$)$. The mean imaging time after $\mathrm{SC}$ injection was 34 min (range, 6-62 min). Of the 5 patients in the control group with no visualized nodes, the average time to imaging was $32 \mathrm{~min}$ (range, 28-44 min). Of the 7 patients in the experimental group with no visualized nodes, the average time to imag-

TABLE 3

Results: Control Versus Experimental Group Pain Experience Based on NIH Pain Scale of 0-10

\begin{tabular}{lccc}
\hline NIH pain scale & All & $\begin{array}{c}\text { Control } \\
\text { group }\end{array}$ & $\begin{array}{c}\text { Experimental } \\
\text { group }\end{array}$ \\
\hline Baseline & & & \\
Range & $0-5$ & $0-1$ & $0-5$ \\
Mean & 0.3 & 0.1 & 0.4 \\
Peak & & & \\
Range & $0-10$ & $1-10$ & $0-9$ \\
\hline Mean & 4.1 & 5.2 & 3.0 \\
Mean increase & +3.8 & +5.1 & +2.6 \\
in pain & & & \\
\hline
\end{tabular}

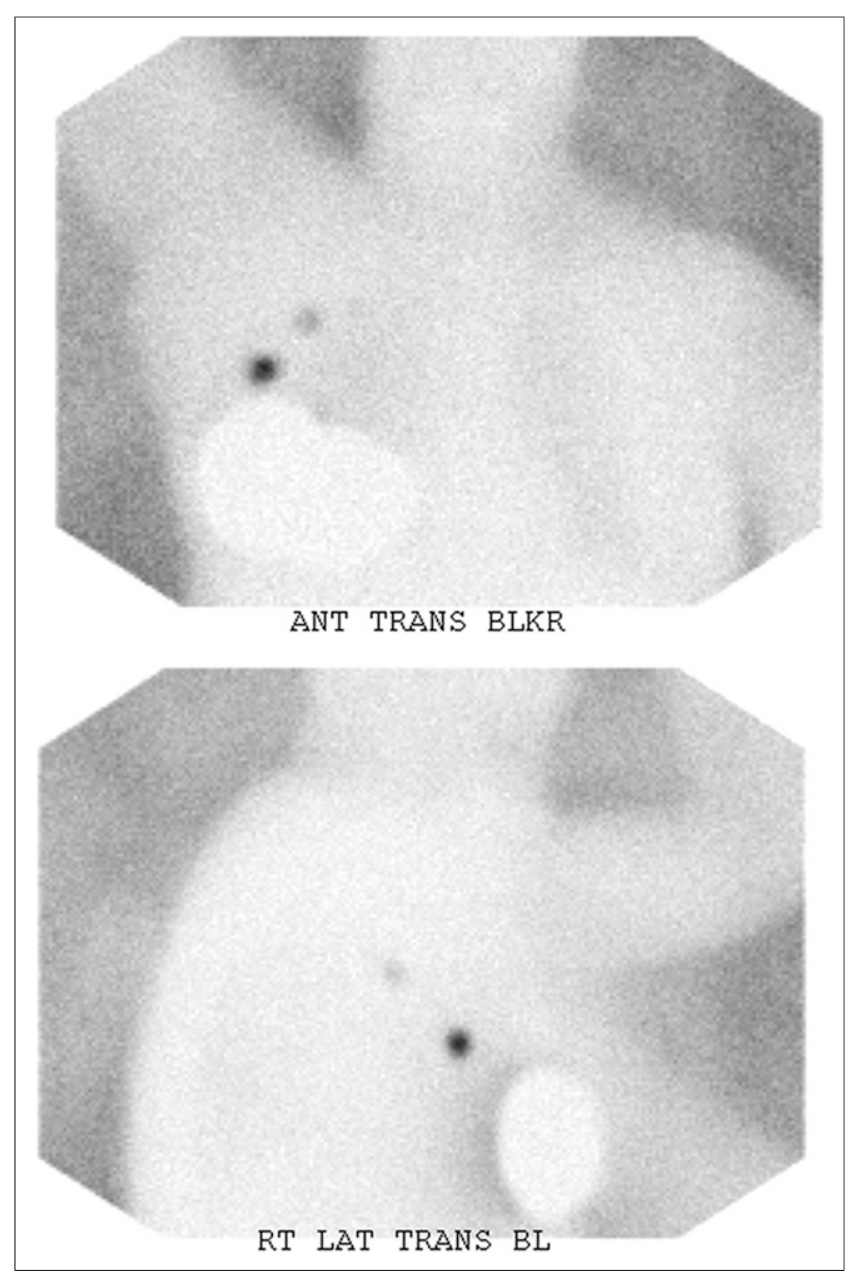

FIGURE 2. Scintigraphic images of chest and axilla in control group. ANT = anterior; $\mathrm{BL}=$ blocker present; $\mathrm{BLKR}=$ blocker present; LAT $=$ lateral; TRANS $=$ transverse.

ing was $39 \mathrm{~min}$ (range, $30-57 \mathrm{~min}$ ). Times between injection and imaging were dependent on the patient's scheduled operating room time, patient arrival time, and daily variations with patient throughput in the nuclear medicine department (Table 4).

\section{DISCUSSION}

It is believed that the pain associated with intradermal SC injection is related to the acidity of the solution and to the focal distention of potential spaces between layers of skin and subcutaneous tissues. Breast lymphoscintigraphy has traditionally been performed without local anesthesia, likely because of the rational assumption that any benefit gained from local anesthesia would be outweighed by the increase in pain associated with additional needle sticks. Several techniques have been used to minimize discomfort during lymphoscintigraphy, which have been described previously in the literature. These techniques include performing the minimal number of injections with the minimal volume of SC, applying topical anesthetic, buffering SC with sodium bicarbonate, and adding both sodium bicarbonate and lidocaine 


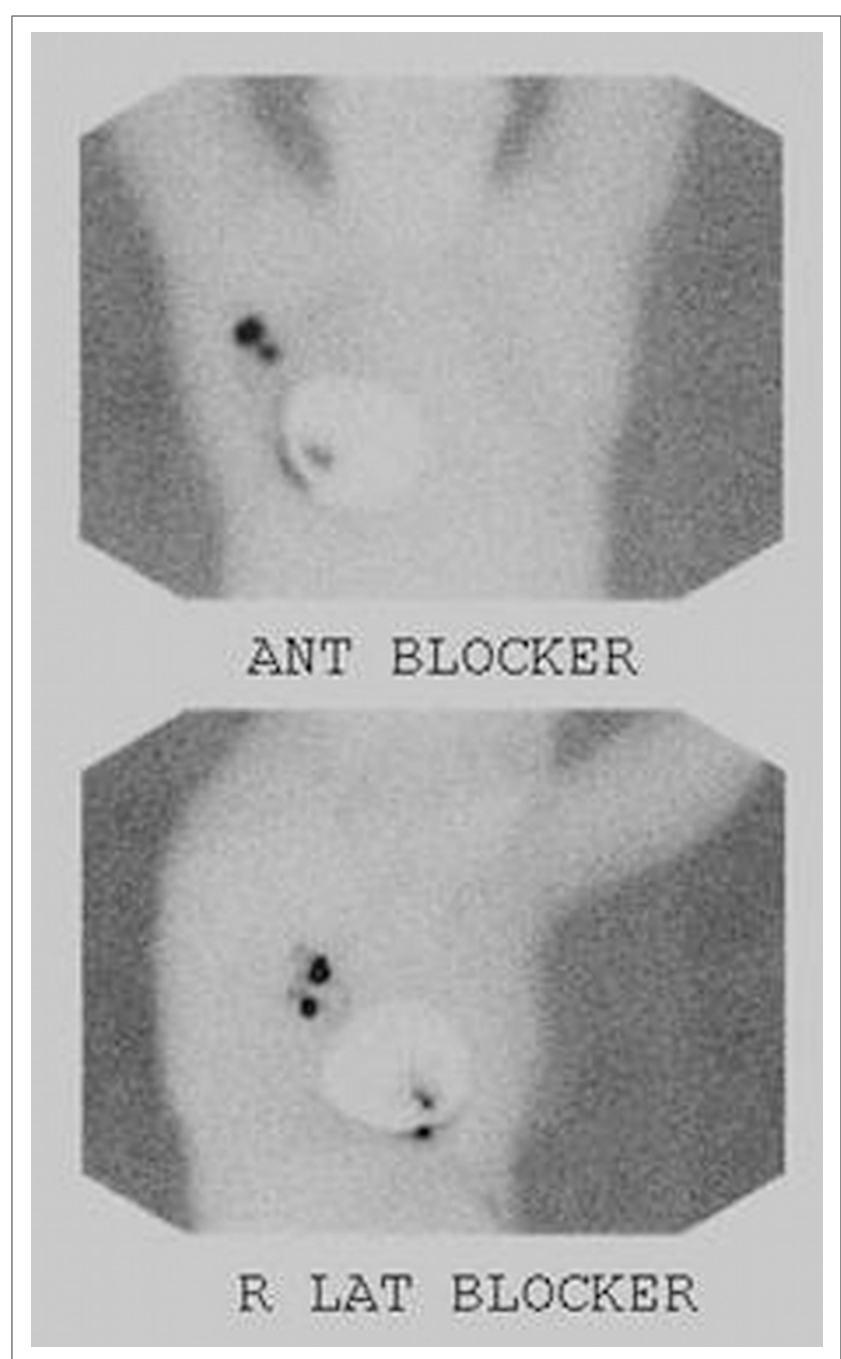

FIGURE 3. Scintigraphic images of chest and axilla in experimental group. ANT = anterior; LAT $=$ lateral.

to the SC before injection. The technique used during this study was a variation of these techniques, and review of the current literature revealed no existing study examining the utility of giving local anesthesia before, and separate from, the SC injection.

Fetzer et al. found that the technique of applying a eutectic mixture of local anesthetics to the skin of the breast $1 \mathrm{~h}$ before injection reduced pain (2). Conversely, Chandler et al. found that there was no significant correlation between reported pain, the use of topical anesthetic cream, the timing of the cream application, the age of the patient, or the breast density (3). O'Connor et al. similarly concluded that applying topical anesthetic to the breast did not lead to a statistically significant reduction in pain scores (4). Although there is some discordance regarding the effectiveness of topical anesthetic between these studies, the application of topical cream universally increased the time required to perform the lymphoscintigraphy procedure by between $20 \mathrm{~min}$ and $1 \mathrm{~h}$. In our experience, patients are typically scheduled for elective breast surgery early in the morning. Any change to our protocol that would increase the length of time that a surgeon would need to wait for a patient would not be considered acceptable. Additionally, it would place an extra burden on the patient to apply the cream correctly before arrival or to arrive early to have the cream applied by medical staff.

In regards to buffering the SC before injection, Stokes et al. and Stojadinovic et al. separately concluded that mixing lidocaine or lidocaine with sodium bicarbonate with the SC reduced pain when compared with placebo and topical anesthetic, without significantly altering the detection of sentinel lymph nodes $(5,6)$. However, altering the SC solution before injection has the potential to change the SC particle size, $\mathrm{pH}$, and possibly the particle aggregation and kinetics through the lymphatics. These effects were studied by Loveless et al., who determined it was possible to add lidocaine or bicarbonate without significantly altering the kinetics, but the mixture works best when a sodium bicarbonate strength of $8.4 \%$ was used to obtain a $\mathrm{pH}$ of 7.1 (7). The stability of a mixture of SC and lidocaine was questioned and studied by Dura et al. who determined that appropriately mixed solutions could be stable for up to $8 \mathrm{~h}$ when stored in the injection syringe (8).

Because mixing lidocaine or sodium bicarbonate with SC decreases patient pain on injection, it seems reasonable to premix the SC before injection. However, many institutions, including our own, are purchasing sealed single doses of radiopharmaceuticals from regional radiopharmacies and do not have the authority or compounding licenses to alter the sealed doses before administration. This approach of buffering the $\mathrm{SC}$ is, therefore, often not an option. For institutions that continue to make their own radiopharmaceuticals, verifying the $\mathrm{pH}$ value while meeting the U.S. Pharmacopeial Convention standards or radiochemical purity for each buffered

TABLE 4

Lymph Node Detection

\begin{tabular}{llcc}
\hline \multicolumn{1}{c}{ Node detection characteristic } & All & Control group & Experimental group \\
\hline Patients with 1 or more axillary node detected & 37 & 19 & 18 \\
Patients without axillary nodes detected & 12 & 5 & 7 \\
Mean no. of axillary nodes detected & 1.2 & 1.2 & 1.3 \\
Imaging time (min) & 34 & 34 & 33 \\
Mean & $6-62$ & $25-62$ & $6-59$ \\
\hline Range & & & \\
\hline
\end{tabular}


dose of SC seemingly would introduce additional steps into the routine protocol.

Using the technique described in this study, we were able to demonstrate a decrease in perceived pain in the experimental group relative to the control group. Given the nearimmediate anesthetic effects of lidocaine, the decrease in pain was accomplished without any significant increase in procedure time. Just as importantly, our technique did not require any alteration of the sealed dose of SC.

Our study also showed that subcutaneous anesthesia with buffered lidocaine before intradermal SC injection did not interfere with lymph node visualization. Although there were patients in the experimental group in whom lymph nodes were not detected, there was no significant difference in detection, compared with the control group. Of the patients with no lymph nodes visualized, the time to imaging was not likely a significant factor, because none of these patients was imaged earlier than $28 \mathrm{~min}$. The etiology for the nonvisualization is uncertain but is likely related to variations in lymphatic drainage and subtle variations in injection techniques.

Before our study, McMasters et al. had shown that intradermal injection of SC improved sentinel lymph node detection and decreased the false-negative rate as compared with peritumoral or subdural injections (9). Additionally, Povoski et al. had shown intradermal injections were superior to intraparenchymal or subareolar injections (10). The use of buffered lidocaine around the intradermal injection sites of ${ }^{99 m} \mathrm{Tc}-\mathrm{SC}$ does not appear to interfere with lymph node detection.

The amount of pain that patients feel during breast lymphoscintigraphy is variable, but some patients report significant pain. Radowsky et al. have shown that providers tend to underestimate the level of pain regardless of the technique used (11). It is incumbent on providers to attempt to reduce the level of pain and discomfort experienced by patients during any medical procedure, including breast lymphoscintigraphy, as long as it does not interfere with the results of the procedure.

\section{CONCLUSION}

The results from our study indicate that injecting subcutaneous buffered lidocaine before intradermal injection of ${ }^{99 m} \mathrm{Tc}-\mathrm{SC}$ for breast lymphoscintigraphy significantly decreases patient pain without interfering with lymph node visualization.

\section{DISCLOSURE}

No potential conflict of interest relevant to this article was reported.

\section{REFERENCES}

1. McCaffery M, Beebe A. Pain: Clinical Manual for Nursing Practice. Baltimore, MD: V. V. Mosby Company; 1993.

2. Fetzer S, Holmes S. Relieving the pain of sentinel lymph node biopsy tracer injection. Clin J Oncol Nurs. 2008;12:668-670.

3. Chandler KJ, Hunt CH, Morreale R, Johnson GB, Peller PJ. Effect of patient age, breast density, and topical anesthetic cream on perceived pain with sentinel lymph node scintigraphy. J Nucl Med Technol. 2012;40:44-47.

4. O'Connor JM, Helmer SD, Osland JS, Cusick TE, Tenofsky PL. Do topical anesthetics reduce periareolar injectional pain before sentinel lymph node biopsy? Am J Surg. 2011;202:707-711.

5. Stokes M, Jansons D, Gilmore D, Donohoe K. Buffered Tc-99m sulfur colloid substantially reduces the pain of lymphoscintigraphy injections [abstract]. $\mathrm{J} \mathrm{Nucl}$ Med. 2008;49(suppl 1):419P.

6. Stojadinovic A, Peoples GE, Jurgens JS, et al. Standard versus $\mathrm{pH}$-adjusted and lidocaine supplemented radiocolloid for patients undergoing sentinel-lymphnode mapping and biopsy for early breast cancer (PASSION-P trial): a double blind, randomized controlled trial. Lancet Oncol. 2009;10:849-854.

7. Loveless VS, Surdock CP, Bhattacharjee H. Evaluation of zeta-potential and particle size of technetium ${ }^{99 \mathrm{~m}} \mathrm{Tc}$-sulfur colloid subsequent to the addition of lidocaine and sodium bicarbonate. J Nucl Med Technol. 2010;38:49-52.

8. Dura JV, Hinkle GH. Stability of a mixture of technetium $99 \mathrm{~m}$ sulfur colloid and lidocaine hydrochloride. Am J Health Syst Pharm. 2007;64:2477-2479.

9. McMasters KM, Wong SI, Martin RC, et al. Dermal injection of radioactive colloid is superior to peritumoral injection for breast cancer sentinel lymph node biopsy: results of a multi-institutional study. Ann Surg. 2001;233:676-687.

10. Povoski SP, Olsen JO, Young DC, et al. Prospective randomized clinical trial comparing intradermal, intraparenchymal and subareolar injection routes for sentinel lymph node mapping and biopsy in breast cancer. Ann Surg Oncol. 2006; 13:1412-1421.

11. Radowsky JS, Baines L, Howard RD, Shriver CD, Buckenmaier CC 3rd, Stojadinovic A. Pain ratings by patients and their providers of radionucleotide injection for breast cancer lymphatic mapping. Pain Med. 2012;13:670-676. 